\section{SM Journal of Nephrology and Therapeutics}

\author{
Article Information \\ Received date: Jun 09, 2016 \\ Accepted date: Jun 10, 2016 \\ Published date: Jun 15, 2016

\section{${ }^{*}$ Corresponding author}

Bhupesh Panwar, University of Alabama at Birmingham, ZRB 522, 1720 2nd AVE S, Birmingham, AL 35294-0006, United States, Tel: 205-975-3304; Fax: 205-9966465; Email: bpanwar@uabmc.edu

Distributed under Creative Commons CC-BY 4.0

Article DOI 10.36876/smjnt.1001

\section{OPEN ACCESS}

\section{Targeting Hepcidin to Treat Anemia of Chronic Kidney Disease}

\author{
Bhupesh Panwar ${ }^{1 \star}$ \\ ${ }^{1}$ Department of Medicine, University of Alabama at Birmingham, Birmingham, United States
}

\section{Abstract}

Treatment of anemia in Chronic Kidney Disease (CKD), a problem of public health proportions, involves utilization of invasive and costly treatment options. Moreover, recent clinical trials have revealed several complications arising from the use of erythropoiesis stimulating agents in the treatment of anemia of CKD. There is an unmet need for safer, cheaper and effective treatment options for anemia of CKD.

Hepcidin, the master regulator of iron trafficking in the body, is known to be elevated in CKD. Since hepcidin effectively blocks iron absorption from the gut and iron release from the cells of the reticuloendothelial system, elevated hepcidin levels lead to a state of iron restricted erythropoiesis. Therefore, as shown in experimental data and animal studies, correcting hepcidin levels can potentially ameliorate anemia of CKD.

Among several known factors contributing to elevated hepcidin levels in CKD, vitamin D deficiency has recently emerged as an independent risk factor. Recent studies have shown that vitamin D can lower hepcidin levels by directly inhibiting hepcidin gene transcription at the nuclear level in the hepatocytes. Since vitamin $D$ treatment is known to be safe and cost effective, it can prove to be an ideal agent to target elevated hepcidin levels thereby correcting the central mechanism that leads to development of anemia in CKD.

\section{Editorial}

Anemia is a common complication of Chronic Kidney Disease (CKD) [1]. Traditionally, the use of Erythropoiesis Stimulating Agents (ESAs), iron infusions and blood transfusions are considered to be the cornerstone therapeutics for the treatment of anemia of CKD. However, these treatment options are not only costly and invasive, use of ESAs has been recently linked to adverse events and poor outcomes in several large scale randomized clinical trials in the CKD population [2-4]. This has recently shifted the focus on finding newer therapeutics that are safer, cost effective and efficacious. Over the last decade, the research in this direction has gained momentum due to the discovery of hepcidin, a novel 25 amino-acid peptide, which is now believed to be the master regulator of iron homeostasis in health and disease [5].

Hepcidin controls iron trafficking in the body by binding to ferroportin- a transmembrane iron channel [6]. Since ferroportin is the only known exit route for intracellular iron, hepcidin blocks cellular iron exit by binding to ferroportin and promoting its degradation and internalization [6]. Thus, by binding to ferroportin on the basolateral membrane of the duodenal enterocytes and on the cell membrane of the reticuloendothelial cells, hepcidin reduces iron absorption and iron release from macrophages, respectively. This leads to decreased iron bio-availability and iron restriction. Since, hepcidin levels are known to be elevated in CKD, [7] hepcidin is now believed to play a central role in mediation of anemia of CKD by reducing iron bio-availability and promoting iron restricted erythropoiesis [8]. Animal studies have demonstrated improvement in iron availability by lowing hepcidin using anti-hepcidin antibodies, providing evidence to suggest that targeting elevated hepcidin in CKD could potentially ameliorate anemia of CKD [9].

In our quest to develop effective anti-hepcidin therapeutics, identifying the reversible underlying causes leading to elevated hepcidin levels in CKD is of paramount importance. In individuals with $\mathrm{CKD}$, hepcidin levels could be elevated due to several reasons of which decreased renal clearance (reduced glomerular filtration rate) is thought to be the primary mechanism. Other possible mechanisms include increase in inflammatory cytokines in CKD and regular iron therapy which stimulate hepcidin synthesis [10]. Several studies have now shown that the factors described above explain only part of the variability of hepcidin concentrations in CKD, indicating that other factors likely play a role in stimulating hepcidin production in CKD $[7,11,12]$. Over the last few years, vitamin D deficiency, which is highly prevalent in CKD, has emerged as a putative factor contributing towards development hepcidin excess in CKD.

A recent cell-line study showed that vitamin D directly inhibits hepcidin transcription in hepatocytes by direct transcriptional suppression of the hepcidin gene leading to decreased hepcidin synthesis [13]. A pilot study in healthy human volunteers showed a $34 \%$ decrease in hepcidin levels within 24 hours after a single large dose of 25D-hydroxy vitamin D [13]. Along the same lines, another study in individuals with CKD revealed an inverse association between change in vitamin 
D levels with vitamin D replacement and serum hepcidin levels [14]. Taken together, these recent findings point towards a potential role of vitamin $\mathrm{D}$ in the treatment of anemias with underlying iron restriction due to elevated hepcidin levels such as anemia of CKD.

Although efficacy of vitamin D for the treatment of anemia of CKD still remains to be proven, it could potentially emerge as an ideal agent to treat anemia of CKD by targeting hepcidin synthesis in that compared to existing treatment modalities, it is relative safer and more cost-effective. Well-designed future physiologic and interventional studies are needed to study the effect of vitamin D replacement on hepcidin levels, iron absorption, iron bio-availability, and hemoglobin levels in CKD.

\section{References}

1. Stauffer ME, Fan T. Prevalence of Anemia in Chronic Kidney Disease in the United States. PLoS One. 2014; 9: e84943.

2. Drueke TB, Locatelli F, Clyne N, Eckardt KU, Macdougall IC, Tsakiris D, et al Normalization of hemoglobin level in patients with chronic kidney disease and anemia. N Engl J Med. 2006; 355: 2071-2084.

3. Pfeffer MA, Burdmann EA, Chen CY, Cooper ME, de Zeeuw D, Eckardt KU et al. A trial of darbepoetin alfa in type 2 diabetes and chronic kidney disease. N Engl J Med. 2009; 361: 2019-2032.

4. Singh AK, Szczech L, Tang KL, Barnhart H, Sapp S, Wolfson M, et al Correction of Anemia with Epoetin Alfa in Chronic Kidney Disease. N Engl J Med. 2006; 355: 2085-2098.

5. Ganz T. Hepcidin, a key regulator of iron metabolism and mediator of anemia of inflammation. Blood. 2003; 102: 783-788.
6. Nemeth E, Tuttle MS, Powelson J, Vaughn MB, Donovan A, Ward DM, et al. Hepcidin regulates cellular iron efflux by binding to ferroportin and inducing its internalization. Science. 2004; 306: 2090-2093.

7. Ashby DR, Gale DP, Busbridge M, Murphy KG, Duncan ND, Cairns TD, et al. Plasma hepcidin levels are elevated but responsive to erythropoietin therapy in renal disease. Kidney Int. 2009; 75: 976-981.

8. Babitt JL, Lin HY. Molecular mechanisms of hepcidin regulation: implications for the anemia of CKD. Am J Kidney Dis. 2010; 55: 726-741.

9. Sasu BJ, Cooke KS, Arvedson TL, Plewa C, Ellison AR, Sheng J, et al Antihepcidin antibody treatment modulates iron metabolism and is effective in a mouse model of inflammation-induced anemia. Blood. 2010; 115: 36163624.

10. Kuragano T, Shimonaka Y, Kida A, Furuta M, Nanami M, Otaki Y, et al. Determinants of hepcidin in patients on maintenance hemodialysis: role of inflammation. Am J Nephrol. 2010; 31: 534-540.

11. Malyszko J, Malyszko JS, Pawlak K, Mysliwiec M. Hepcidin, iron status, and renal function in chronic renal failure, kidney transplantation, and hemodialysis. Am J Hematol. 2006; 81: 832-837.

12. Uehata T, Tomosugi N, Shoji T, Sakaguchi $Y$, Suzuki A, Kaneko T, et al. Serum hepcidin-25 levels and anemia in non-dialysis chronic kidney disease patients: a cross-sectional study. Nephrol Dial Transplant. 2012; 27: 10761083.

13. Bacchetta J, Zaritsky JJ, Sea JL, Chun RF, Lisse TS, Zavala K, et al. Suppression of iron-regulatory hepcidin by vitamin D. J Am Soc Nephrol. 2014; 25: 564-572.

14. Zughaier SM, Alvarez JA, Sloan JH, Konrad RJ, Tangpricha V. The role of vitamin $\mathrm{D}$ in regulating the iron-hepcidin-ferroportin axis in monocytes. $\mathrm{J}$ Clin Transl Endocrinol. 2014; 1: 19-25. 